Original paper

\title{
Expression of intrahepatic CD3, CD4, and CD8 T cells in biliary atresia
}

\author{
Behairy E. Behairy ${ }^{1}$, Nermine Ehsan' ${ }^{1}$ Magdy Anwer ${ }^{1}$, Alif Allam¹, Ibramem El-Henawy' ${ }^{1}$ Nesreen Abdel Hameed', \\ Haidy M. Zakaria² \\ ${ }^{1}$ National Liver Institute, Shebeen El-Kom, Egypt \\ ${ }^{2}$ Quesna Central Hospital, Quesna, Egypt
}

\begin{abstract}
Aim of the study: Assessment of the expression of cluster of differentiation (CD)3, CD4, and CD8 T cells in biliary atresia (BA) cases in comparison to neonatal cholestasis other than BA.

Material and methods: This study included 79 patients: 34 patients with BA (BA group) and 35 patients with neonatal cholestasis due to causes other than BA (cholestasis group), and 10 normal liver donor as a control group. Immunohistochemical staining or CD3, CD4, and CD8 T cells in liver tissues for the 3 groups were evaluated.

Results: Presence of clay stool, high gamma-glutamyl transferase levels, thrombocytosis, and non-contractibility of the gallbladder was the main clinical, laboratory, and radiological findings, distinguishing BA from other disorders causing neonatal cholestasis. Portal ductular proliferation, bile plugs in portal ductules, and advanced grades of fibrosis were more predominant in liver biopsy specimens of BA patients. The CD3+, CD4+, and CD8+ expression in patients with BA were significantly higher than in both cholestasis and control groups, while it was comparable in the cholestasis and control groups, with cutoff values of 25,12 , and 2.5 cells/portal tract, respectively, differentiating between $B A$ and cholestatic patients.

Conclusions: Immune-mediated destruction of bile ducts is incriminated in the pathogenesis of BA. Lymphocytic infiltrate in portal tract is primarily composed of $C D 3, C D 4$, and $C D 8 T$ cells. Immunostaining of liver tissue for $\mathrm{CD} 3, \mathrm{CD} 4$, and CD8 T cells can help in ensuring diagnosis of BA.
\end{abstract}

Key words: cholestasis, biliary atresia, cluster of differentiation.

\section{Address for correspondence}

Dr. Haidy M. Zakaria, Quesna Central Hospital, 20048 Quesna, Menofyia, Egypt, phone: +2 01005768306 ,

e-mail: drhaydi2000@gmail.com

\section{Introduction}

Biliary atresia (BA) is the most significant lifethreatening hepatobiliary disorder in children, which results from destructive inflammatory process of both intrahepatic and extrahepatic bile ducts. If untreated, progressive liver cirrhosis leads to death by 2 years of age. Diagnosis of BA is still difficult, and operative cholangiography to visualize the biliary tract is still the golden standard for the diagnosis. Kasai portoenterostomy is the first choice of treatment and good results depend on early diagnosis and operation [1].
The immune response focused within the portal tracts in BA is not present in other similar neonatal cholestatic disorders. This unique immune pattern suggests that the observed immune repertoire is not merely a stereotypic response to the accumulation of biliary constituents in the neonatal cholestatic liver, but rather an indicator of specific immune processes involved in the pathogenesis of BA. Further elucidation of the nature of immune response in the biliary tree of these patients will be essential for advancing of our understanding of this disorder, and developing new strategies towards an effective treatment [2]. 
The inflammatory milieu presented in the portal tracts consists of Kupffer cells, cluster of differentiation (CD)4+ $\mathrm{T}$ cells, and natural killer (NK) cells. However, controversy persists as to the cause of this portal tract inflammation, and it remains unclear whether BA is a primary immune-mediated disease, or the inflammation is a secondary response to bile stasis and biliary obstruction [3].

The aim of this work is to assess the expression of $\mathrm{CD} 3+, \mathrm{CD} 4+$, and CD8+ T cells in BA cases in comparison to neonatal cholestasis other than BA.

\section{Material and methods}

The study included 79 patients recruited from the inpatient of Department of Pediatric Hepatology, National Liver Institute, Menoufia University.

All patients were subjected to full history taking, thorough clinical examination, and investigations such as: liver functions, prothrombin concentration, complete blood count, viral and toxoplasma markers, as well as specific investigations according to the suspected etiology. Abdominal ultrasound (US) was done to assess the size and echo-pattern of the liver, the size of the spleen, the presence of ascites, gallbladder (GB) size, and contractility. Liver biopsy was completed for histopathological assessment.

Definitive diagnosis of BA was confirmed by intraoperative cholangiogram. However, surgical cholangiography is typically performed by injecting contrast material through the gallbladder. If no communication is seen between the biliary tree and the gastrointestinal tract, $\mathrm{BA}$ is diagnosed.

After confirming the final diagnosis, patients were divided into two groups: BA group (34 patients) and cholestasis group (35 patients with cholestasis due to causes other than BA). Ten healthy liver donors served as control group.

The non-BA cholestasis groups included 10 patients with familial intrahepatic cholestasis, 13 patients with metabolic liver disease ( 8 patients with galactosemia, 2 patients with tyrosinemia, 3 patients with NiemannPick type C), 6 patients with idiopathic neonatal hepatitis, and a miscellaneous group included 6 patients (solitary cases of CMV hepatitis, congenital hepatic fibrosis, choledochal cyst, Alagille syndrome, bile acid synthetic defects, and arthrogryposis-renal dysfunction-cholestasis [ARC] syndrome).

\section{Immunohistochemical staining of liver tissue and histopathological evaluation}

Paraffin-embedded, formalin-fixed liver tissues of all studied cases were cut into three different $4-\mu \mathrm{m}$ thick sections and placed on positive charged slides. Each paraffin section was deparaffinized in xylene and rehydrated through a graded series of ethanol. The antigen retrieval was performed by steaming the slides in appropriate buffer at different temperatures. Endogenous peroxidase was blocked using a $3 \% \mathrm{H}_{2} \mathrm{O}_{2}$ methanol solution. The immunostaining technique applied in this study was the improved horseradish peroxidase amplified system, CD3, CD4, and CD8 monoclonal antibody (Thermo Scientific, Lab Vision Corporation, Fremont, CA 945386406 , USA). In this system, three components were utilized: the primary antibody specific for the antigen to be localized, the secondary anti-immunoglobulin, which is capable of binding both of the primary antibody, and the horseradish peroxidase enzyme complex. Finally, the reaction can be visualized by a substrate/chromogen reagent, which is called diaminobenzidine (DAB).

Paraffin sections were cut by microtome at $4-\mu \mathrm{m}$ thickness, and mounted on the Superfrost glass slides. The sections were deparaffinized in 2 successive rinse of xylene.

The deparaffinized sections were re-hydrated by immersing them in absolute $100 \%$ ethanol for $10 \mathrm{~min}$; then in $95 \%$ ethanol for $5 \mathrm{~min}$, and then in $70 \%$ ethanol for $5 \mathrm{~min}$. Finally, they were immersed in distilled water for $5 \mathrm{~min}$. Between each step, excess liquid was drained. Afterwords, the sections were put twice in phosphate buffer saline PBS for $5 \mathrm{~min}$ each. To reduce non-specific background staining due to endogenous peroxidase, the sections were incubated in 3\% hydrogen peroxide for $15 \mathrm{~min}$ in humidity chamber (SigmaAldrich, CAS:7722-84-1). Then, the sections were rinsed in PBS twice for 5 min each section. For antigen retrieval, heat induced epitope retrieval (HIER) procedure was used. It is used to reverse the loss of antigenicity that occurs with some epitopes in formalin-fixed paraffin embedded tissues. The slides were washed in distilled water 3 times, 2 min each. Ultra V block was applied, and slides were incubated for $5 \mathrm{~min}$ in humidity chamber.

Each primary antibody was applied on a separated tissue section. The slides were incubated horizontally in a humidity chamber for $60 \mathrm{~min}$ at room temperature. After 2-3 washes, incubation of secondary antibody for $30 \mathrm{~min}$ at room temperature was applied (Histostain $^{\circledR}$-Plus broad spectrum, Invitrogen ${ }^{\mathrm{TM}}$ ). After 2-3 washes, horseradish peroxidase (Histostain ${ }^{\circledR}$-Plus broad spectrum, Invitrogen ${ }^{\mathrm{TM}}$ ) was subsequently performed. Visualization of the reaction was created with diaminobenzidine DAB (GEEMED with REF:5400172), and counterstaining with hematoxylin. Negative control staining was performed with cold PBS, instead of the primary antibody. 


\section{Interpretation of results}

The revised technique involved a semi-quantitative assessment of immunostaining expression. Positive immunostaining was identified by brownish discoloration of the cytoplasm and/or cell membrane. T cells (CD3, CD4, and CD8) were either positive or negative for immunoreaction with no intensity variation. Biopsy samples were considered adequate for the purposes of this study if at least 3 complete portal tracts were present. The semi-quantitative assessment included evaluation of the average positive staining cells among inflammatory infiltrate in portal tracts at high-power fields of view $(\times 400)$ using Olympus light microscope. For each biopsy, an average of immunoreactive (positive) cells counted in 3 different portal tracts along the length of tissue core biopsy was calculated. Subsequently, all cells morphologically consistent with T-cells showing unequivocal immunohistochemical expression of $\mathrm{CD} 3, \mathrm{CD} 4$, or $\mathrm{CD} 8$ were counted. The percentage of positive cells among inflammatory infiltrate in portal tracts for each studied antibody was assessed. Then, an average of count of the frequency of each positive staining on the total liver inflammatory cells was calculated. The evaluating pathologist was unaware of the clinical diagnosis of the patient associated with any of the tissue sections at the time of analysis. Immunohistochemical results were statistically correlated with the scoring system applied for BA.

\section{Statistical analysis}

Descriptive results were expressed as mean \pm standard deviation (mean $\pm \mathrm{SD}$ ), median $\left(25^{\text {th }}-75^{\text {th }}\right)$ or number (percentage) according to the nature of the data. For quantitative data, statistical significance among all groups was tested by Kruskal-Wallis non-parametric test. Significance between individual groups was tested by applying Mann-Whitney $U$ test. Multiple comparisons between each individual group versus all the other groups were tested using Post Hoc LSD (least significant difference) test. For qualitative data, significance between groups was tested by $\chi^{2}$ test or Fisher's exact test. Correlation was tested by Spearman's test. Results were consider significant if $p$-value $\leq 0.05$. The diagnostic performance of immunohistochemistry scores were measured as sensitivity, specificity, positive predictive value (PPV), negative predictive value (NPV), and all were expressed as percentages. The cutoff for optimal clinical performance was determined from the receiver operating characteristic (ROC) curve. Statistical analysis was done using SPSS statistical package, version 18 (SPSS Inc., Chicago, IL, USA).

\section{Ethics committee approval}

The study was approved by the Research Ethics Committee of the National Liver Institute, Menoufia University, Egypt, and was in accordance with the Helsinki Declaration from 1975.

\section{Results}

This study included 79 patients: 34 patients with BA (BA group) and 35 patients with neonatal cholestasis due to causes other than BA (cholestasis group), and 10 healthy liver donor as a control group. Of the BA group, 18 patients were females and 16 were males; of the cholestasis group, 15 patients were females and 20 were males. In the control group, there were 4 were females and 6 males. The mean age in the control group (322.9 \pm 126.03 months) was significantly higher than that in the cholestasis group $(3.2 \pm 3.63$ months) and BA group (1.79 \pm 0.57 months), with a $p$-value $<0.0001$.

This study showed predominance of negative consanguinity and family history in BA group, with 79.4\% and $97.1 \%$ incidence, respectively ( $p$-value $<0.05$ ). Clinically, clay stool was found in $100 \%$ of patients in BA group, and in $34.3 \%$ of patients in the cholestasis group, with $p$-value $<0.0001$. The gamma-glutamyl transferase (GGT) and platelets count were significantly higher in BA than in non-BA cholestasis group. On US, presence of abnormal GB was significantly higher in BA group (100\%) in comparison to $38 \%$ of the non-BA infants $(p<0.0001)$ (Table 1$)$. However, the presence of hepatomegaly, splenomegaly, and ascites were comparable between groups $(p>0.05)$.

Portal ductular proliferation, bile plugs in portal ductules, and advanced grades of fibrosis were more predominant in liver biopsy specimens of BA patients (Table 1). The Lee and Loi histopathological score was significantly higher in BA $(8.65 \pm 3.06)$ than in nonBA $(4.69 \pm 1.92)$.

The median CD3, CD4, and CD8 cell counts in patients with BA were significantly higher than that in both cholestasis and control groups, while it was comparable in the cholestasis and control groups (Table 2 and Fig. 1), with cutoff values off 25,12 , and 2.5 cells/ portal tract, respectively, differentiating between BA and cholestatic patients (Fig. 2).

\section{Discussion}

Biliary atresia is an obstructive cholangiopathy of unknown origin, occurring in the perinatal period. Untreated, neonatal cholestasis leads to liver fibrosis 
Table 1. Comparison of laboratory and histopathological findings between the biliary atresia (BA) and cholestasis groups

\begin{tabular}{|c|c|c|c|}
\hline & $\mathrm{BA}, n=34$ & Cholestasis, $n=35$ & $p$-value \\
\hline \multicolumn{4}{|l|}{ Laboratory parameters } \\
\hline $\mathrm{TB}(\mathrm{mg} / \mathrm{dl})$ & $11.718 \pm 4.3$ & $10.203 \pm 5.17$ & 0.157 \\
\hline $\mathrm{DB}(\mathrm{mg} / \mathrm{dl})$ & $8.600 \pm 3.1$ & $7.051 \pm 3.6$ & 0.110 \\
\hline $\mathrm{ALT}(\mathrm{U} / \mathrm{I})$ & $301.03 \pm 222$ & $301.97 \pm 287.3$ & 0.401 \\
\hline AST (U/I) & $203.94 \pm 193$ & $194.91 \pm 217.1$ & 0.614 \\
\hline $\operatorname{ALP}(U / I)$ & $456.41 \pm 216$ & $477.43 \pm 330.6$ & 0.710 \\
\hline GGT (U/I) & $941.26 \pm 624.4$ & $297.43 \pm 435.2$ & $<0.0001$ \\
\hline Total protein (gm/dl) & $5.144 \pm 0.86$ & $5.040 \pm 0.89$ & 0.622 \\
\hline Alb (gm/dl) & $3.185 \pm 0.5$ & $3.117 \pm 0.66$ & 0.639 \\
\hline PT (seconds) & $12.565 \pm 1.5$ & $12.951 \pm 1.7$ & 0.319 \\
\hline INR & $1.05 \pm 0.12$ & $1.08 \pm 0.14$ & 0.272 \\
\hline Urea (mg/dl) & $15.97 \pm 6.3$ & $17.01 \pm 7.3$ & 0.626 \\
\hline Creatinine (mg/dl) & $0.3 \pm 0.13$ & $0.34 \pm 0.19$ & 0.545 \\
\hline $\mathrm{Hb}(\mathrm{gm} / \mathrm{dl})$ & $10.15 \pm 1.2$ & $9.87 \pm 1.61$ & 0.380 \\
\hline $\mathrm{TLC}\left(\times 10^{3} / \mathrm{mm}^{3}\right)$ & $10.2 \pm 4.8$ & $10.723 \pm 3.2$ & 0.827 \\
\hline Platelets $\left(\times 10^{3} / \mathrm{mm}^{3}\right)$ & $470.4 \pm 199.4$ & $323.4 \pm 183.2$ & 0.004 \\
\hline \multicolumn{4}{|l|}{ Histopathological finding } \\
\hline Intrahepatic cholestasis & $34(100 \%)$ & $29(82.9 \%)$ & 0.125 \\
\hline Ductular proliferation & $33(97.1 \%)$ & $11(31.4 \%)$ & $<0.0001$ \\
\hline Bile plugs & $28(82.4 \%)$ & $12(34.3 \%)$ & $<0.0001$ \\
\hline Giant cells & $7(20.6 \%)$ & $16(45.7 \%)$ & 0.027 \\
\hline Steatosis & $1(2.9 \%)$ & $8(22.9 \%)$ & 0.028 \\
\hline \multicolumn{4}{|l|}{ Grading of fibrosis } \\
\hline No fibrosis & $0(0 \%)$ & $2(5.7 \%)$ & \\
\hline Mild & $9(26.5 \%)$ & 22 (62.9\%) & \\
\hline Moderate & $16(47.1 \%)$ & $7(20.0 \%)$ & \\
\hline Severe & $9(26.5 \%)$ & $4(11.4 \%)$ & 0.005 \\
\hline
\end{tabular}

$T B$ - total bilirubin, $D B$ - direct bilirubin, ALT - alanine aminotransferase, $A S T$ - aspartate aminotransferase, ALP - alkaline phosphatase, GGT - gamma-glutamyl transferase, $A L B$ - albumin, $P T$ - prothrombin time, INR - international normalized ratio, $\mathrm{Hb}$ hemoglobin, TLC - total leukocyte count

and cirrhosis in the first months of life, and death in the first years [4].

The present study revealed that presence of clay stool, high GGT levels, thrombocytosis, and non-contractibil- ity of the gallbladder were the main clinical, laboratory, and radiological findings distinguishing BA from other disorders causing neonatal cholestasis. In agreement with our results, Lee and Chai reported that clay stool was found in $83 \%$ of BA group, while present in 36\% of cholestatic patient, hepatomegaly was equally common in both groups of patients (BA $100 \%$ vs. non-BA $94 \%)$. Also, they reported that splenomegaly was found in nearly equal percentage (BA $51 \%$ vs. non-BA $52 \%$, $p=0.13$ ), ascites were uncommon (3\%) for BA group and $7 \%$ for cholestatic [5]. Moreover, Tang et al. reported that GGT levels were significantly higher in BA patients than those in neonatal cholestasis [6].

Liver biopsy is a cornerstone of the diagnostic work-up of infants with cholestatic jaundice, and it is a standard practice in most pediatric centers to obtain a percutaneous liver biopsy before surgical intervention [7].

Our data showed predominance of ductular proliferation, bile plugs, and advanced grades of fibrosis in other causes of cholestasis. Similar to these results, Rastogi et al. reported that ductular proliferation was the most important histopathological feature in distinguishing BA from other disorders causing neonatal cholestasis, with $p$-value $=0.0002$ [8].

The CD3, CD4, and CD8 expression by immunohistochemical staining was assessed in liver biopsies of patients with BA and cholestasis due to causes other than BA in comparison to healthy controls. Liver biopsies of BA infants showed increased expression of $\mathrm{CD} 3, \mathrm{CD} 4$, and $\mathrm{CD} 8$ cells than that in cholestasis and control groups, with cutoff values off 25,12 , and $2.5 \mathrm{cell} /$ portal tract, respectively, differentiating between BA and cholestatic patients. In agreement with our results, Mack et al. reported that there is an increase in CD3, CD4, and CD8 T cells in the portal tracts of patients with BA compared with normal livers and the cholestatic disease controls, with $p$-value $<0.0001$ [3]. In addition, Shinkai et al. reported that infiltrating CD3+ and CD8+ lymphocytes in the portal tracts were significantly higher in B.A group (3.1 $\pm 0.4,2.8 \pm 0.4)$ compared with cholestatic group (1.7 $\pm 0.3,1.5 \pm 0.5$ ) [9].

Table 2. The mean of $\mathrm{CD} 3, \mathrm{CD} 4$, and $\mathrm{CD} 8$ in patients with biliary atresia (BA), cholestasis, and control group

\begin{tabular}{lcccccc}
\hline Marker & $\begin{array}{c}\text { BA, } \boldsymbol{n}=34 \text {; median } \\
\left(25^{\text {th }}-75^{\text {th }}\right)\end{array}$ & $\begin{array}{c}\text { Cholestasis, } \boldsymbol{n}=35 ; \\
\text { median }\left(25^{\text {th }}-7^{\text {th }}\right)\end{array}$ & $\begin{array}{c}\text { Control, } \boldsymbol{n}=10, \text { median } \\
\left(25^{\text {th }}-75^{\text {th }}\right)\end{array}$ & P1 & P2 & P3 \\
\hline CD3 (cell/portal tract) & $41(27-56)$ & $13(7-20)$ & $6(1-13)$ & $<0.0001$ & $<0.0001$ & 0.152 \\
\hline CD4 (cell/portal tract) & $29(16-44)$ & $7(3-11)$ & $5(2-9)$ & $<0.0001$ & $<0.0001$ & 0.159 \\
\hline CD8 (cell/portal tract) & $14(7-22)$ & $2(1-3)$ & $2(1-4)$ & $<0.0001$ & $<0.0001$ & 0.377 \\
\hline
\end{tabular}

$C D$ - cluster of differentiation 

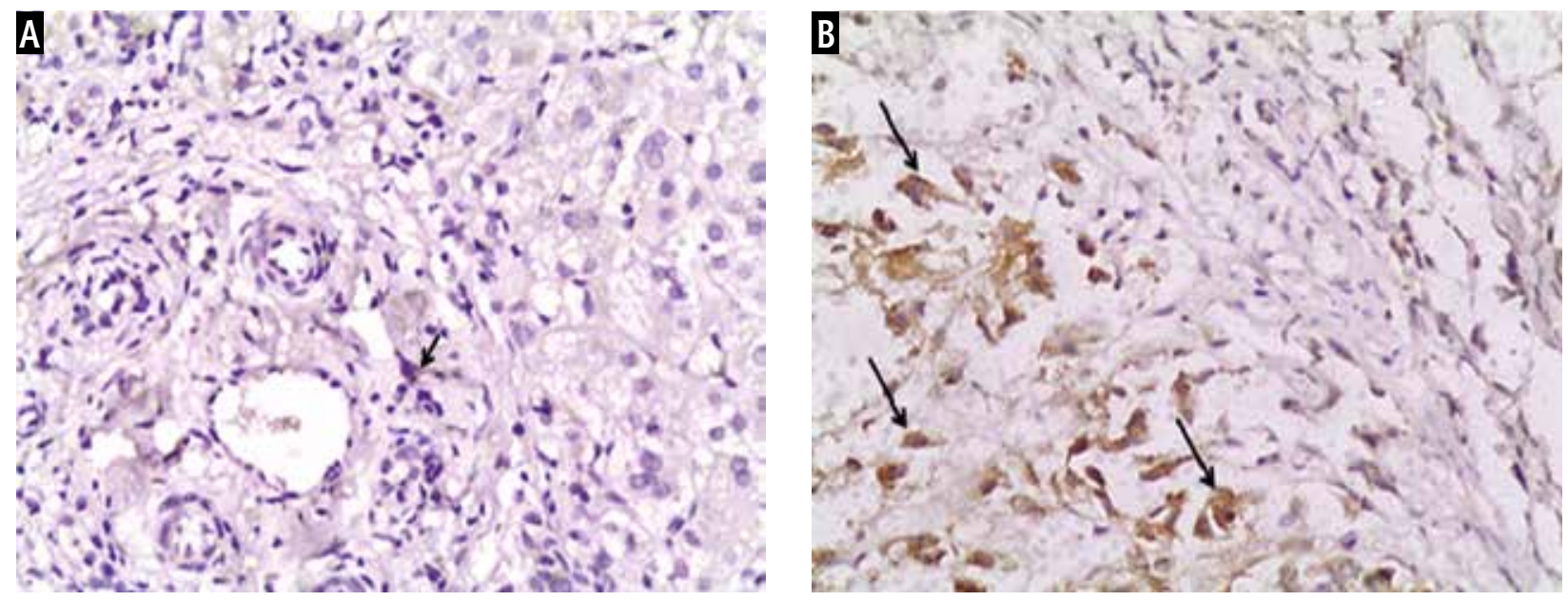

Fig. 1. CD4 cells expression in biliary atresia (BA) and cholestasis groups
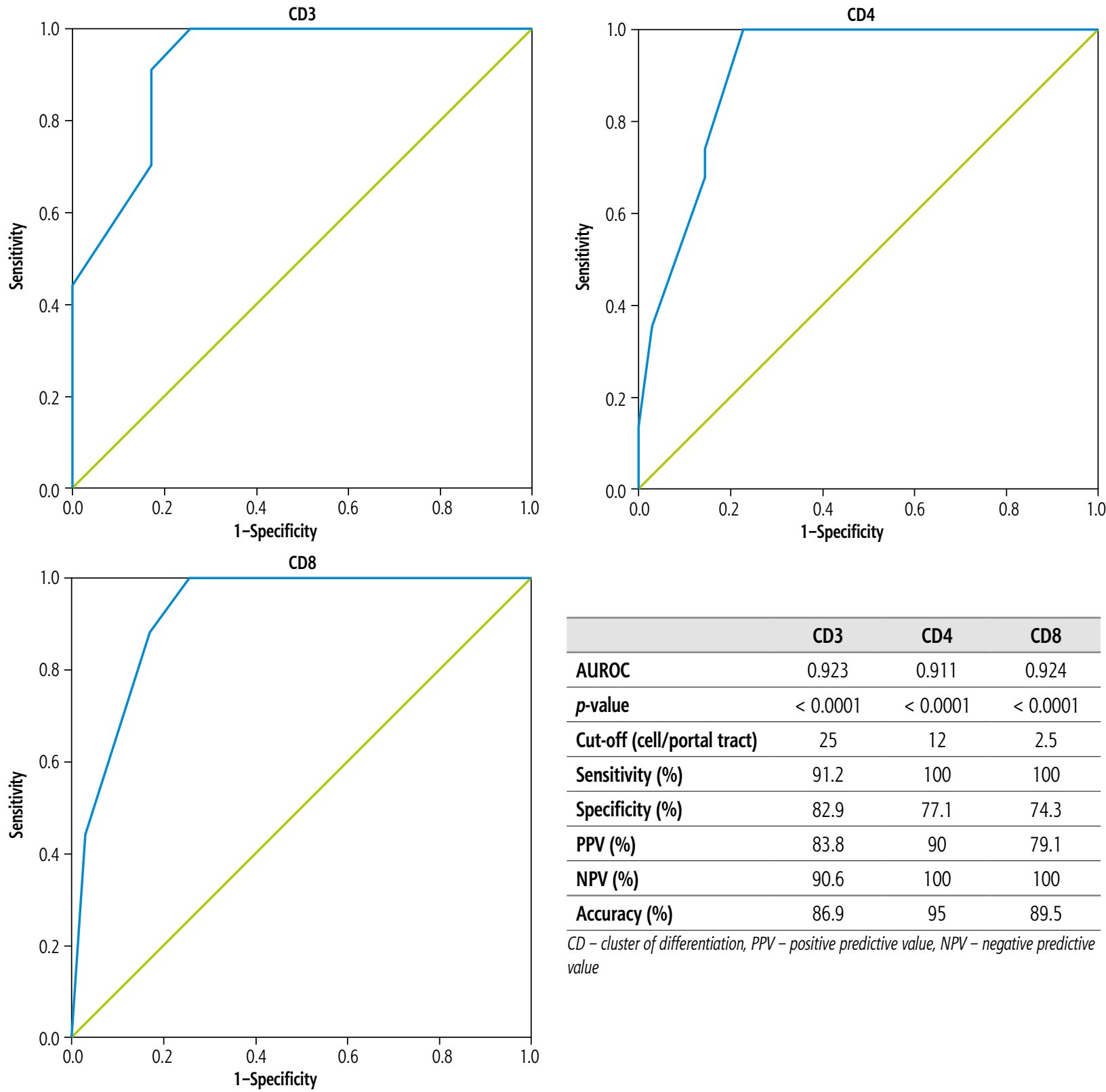

\begin{tabular}{lccc}
\hline & CD3 & CD4 & CD8 \\
\hline AUROC & 0.923 & 0.911 & 0.924 \\
\hline$p$-value & $<0.0001$ & $<0.0001$ & $<0.0001$ \\
\hline Cut-off (cell/portal tract) & 25 & 12 & 2.5 \\
\hline Sensitivity (\%) & 91.2 & 100 & 100 \\
\hline Specificity (\%) & 82.9 & 77.1 & 74.3 \\
\hline PPV (\%) & 83.8 & 90 & 79.1 \\
\hline NPV (\%) & 90.6 & 100 & 100 \\
\hline Accuracy (\%) & 86.9 & 95 & 89.5 \\
\hline
\end{tabular}

$\overline{C D}$ - cluster of differentiation, PPV - positive predictive value, NPV - negative predictive value

Fig. 2. Clinical performance of $C D 3, C D 4$, and $C D 8$ in discriminating between biliary atresia (BA) and cholestasis groups 
Our findings support the role of immune-mediated destruction of bile ducts within the liver of patients with BA. From our study, it can be inferred further that this lymphocytic infiltrate is primarily composed of CD3, CD4+, and CD8+ T cells. Immunostaining of liver tissue for $\mathrm{CD} 3, \mathrm{CD} 4+$, and $\mathrm{CD} 8+\mathrm{T}$ cells can help in ensuring diagnosis of BA. Further investigation will be needed to determine whether the lymphocytes are clonal and reactive to a specific antigen (e.g. viral or self-bile duct epithelial antigen), and the nature of the antigenic epitopes. The findings in this study may shed light on the potential therapeutic use of immunosuppressive therapy in the treatment of BA.

In conclusion, immune-mediated destruction of bile ducts is incriminated in the pathogenesis of BA. Lymphocytic infiltrate in portal tract is primarily composed of CD3, CD4+, and CD8+ T cells. Immunostaining of liver tissue for CD3, CD4+, and CD8+ T cells can help in ensuring diagnosis of BA.

\section{Disclosure}

Authors report no conflict of interest.

\section{References}

1. Agin M, Tumgor G, Alkan M, et al. Clues to the diagnosis of biliary atresia in neonatal cholestasis. Turk J Gastroenterol 2016; 27: 37-41.

2. Sokol RJ, Mack C. Etiopathogenesis of biliary atresia. Semin Liver Dis 2001; 21: 517-524.

3. Mack CL, Tucker RM, Sokol RJ, et al. Biliary atresia is associated with CD4+ Th1 cell-mediated portal tract inflammation. Pediatr Res 2004; 56: 79-87.

4. Chardot C, Buet C, Serinet MO, et al. Improving outcomes of biliary atresia: French national series 1986-2009. J Hepatol 2013; 58: 1209-1217.

5. Lee WS, Chai PF. Clinical features differentiating biliary atresia from other causes of neonatal cholestasis. Ann Acad Med Singapore 2010; 39: 648-654.

6. Tang KS, Huang LT, Huang YH, et al. Gamma-glutamyl transferase in the diagnosis of biliary atresia. Acta Paediatr Taiwan 2007; 48: 196-200.

7. Shneider BL, Brown MB, Haber B, et al. A multicenter study of the outcome of biliary atresia in the United States, 1997 to 2000. J Pediatr 2006; 148: 467-474.

8. Rastogi A, Krishnani N, Yachha SK, et al. Histopathological features and accuracy for diagnosing biliary atresia by prelaparotomy liver biopsy in developing countries. J Gastroenterol Hepatol 2009; 24: 97-102.

9. Shinkai M, Shinkai T, Puri P, et al. Increased CXCR3 expression associated with CD3-positive lymphocytes in the liver and biliary remnant in biliary atresia. J Pediatr Surg 2006; 41: 950-954. 